\title{
INTUSSUSCEPÇÃO EM CÃES: revisão de literatura
}

\author{
A review of intussusception in dogs
}

\author{
Leda Marques de Oliveira-Barros ${ }^{[a]}$, Julia Maria Matera ${ }^{[\mathrm{b}]}$ \\ [a] Pós-Graduanda do departamento de cirurgia da Universidade de São Paulo (FMVZ/USP), São Paulo, SP - Brasil, e-mail: \\ ledamobarros@usp.br \\ ${ }^{[b]}$ Professora titular do departamento de cirurgia da Universidade de São Paulo (FMVZ/USP), São Paulo, SP - e-mail: \\ materajm@usp.br
}

\section{Resumo}

Dentre as alterações obstrutivas do aparelho gastrointestinal de pequenos animais, a intussuscepção merece especial atenção, uma vez que apresenta ocorrência elevada e necessidade de tratamento urgente. A porção íleo-cólica é a mais acometida. Não existe predisposição racial e sexual, porém animais jovens apresentam maior incidência. Fatores predisponentes são frequentemente associados, como parasitismo, gastroenterites e corpos estranhos. A palpação abdominal e o exame ultrassonográfico são importantes ferramentas para o diagnóstico. Para o tratamento, a intervenção cirúrgica se faz necessária, empregando-se técnicas de redução manual ou enterectomia e enteroanastomose. Recorrência do quadro, íleo paralítico, deiscência da anastomose e obstrução intestinal são complicações relacionadas à enfermidade.

Palavras-chave: Cão. Intussuscepção. Cirurgia gastrointestinal.

\begin{abstract}
Among all obstructive disorders of the small animal's gastrointestinal tract, intussusception deserves special attention because it's high occurrence and needs of urgent treatment. The ileum-colic segment is the most overtaken portion of the intestine. There is no sexual and breed predisposing, but young animals can display higher incidence of cases of intussusceptions. Predisposing factors including parasitism, gastroenteritis and foreign bodies are frequently associated to it. Abdominal palpation and ultrasound examination are important tools for diagnosis. To treat intussusceptions, surgical procedures must be necessary and involve manual reduction or enterectomy and enteroanastomosis. Recurrence of an intussusception, paralytic ileus, dehiscence of the anastomosis and intestinal obstruction are complications related to this disease.
\end{abstract}

Keywords: Dog. Intussusception. Gastrointestinal surgery. 


\section{DEFINIÇÃO}

Intussuscepção é definida como a invaginação de uma porção do aparelho gastrointestinal, chamada de intussusceptum, sobre o lúmen da porção adjacente, denominada intussuscepiens (HEDLUNG, 2002; MACPHAIL, 2002; LAMB; MANTIS, 1998). Frequentemente é observada na porção normógrada, ou seja, no sentido peristáltico, porém, existem relatos da recém ocorrência na direção retrograda ou também denominada de oral (APPLEWHITE; HAWTHORNE; CORNELL, 2001; LEWIS; ELLISON, 1987; PATSIKAS et al., 2005). Outra classificação passível de ser utilizada é quanto ao número de intussuscepções, podendo estas serem únicas, múltiplas ou compostas (LEWIS; ELLISON, 1987).

\section{FISIOPATOLOGIA}

A fisiopatologia da intussuscepção ainda não está totalmente elucidada, porém, sugere-se estar relacionada a alterações da atividade motora nos segmentos acometidos, o que resultaria na descontinuidade intestinal, com porções apresentando maior flacidez ou rigidez em comparação às adjacentes. As maiores consequências citadas da intussuscepção são obstrução parcial ou total, isquemia, necrose e ruptura intestinal (LEVITT; BAUER, 1992). Normalmente assume forma curva e de extensão limitada pela tensão exercida pelo mesentério da porção internalizada. Segundo Barker, Van Dreumal e Palmer (1993), o tamanho limite observado em cães é de aproximadamente 12 centímetros.

Eventos desencadeados pelo processo de invaginação intestinal podem ser classificados quanto ao curso da doença. Inicialmente ocorre estrangulamento do retorno venoso com dilatação e congestão. Estágios mais avançados apresentam exsudação de fibrina da superfície serosa, responsável pela aderência intestinal. A obstrução permanente dos vasos está relacionada com isquemia e necrose, fatores determinantes para o desenvolvimento de perfurações e rupturas intestinais tardias (PATSIKAS et al., 2005).

\section{EPIDEMIOLOGIA}

Todas as porções do intestino podem ser acometidas, entretanto, observa-se maior incidência no segmento ileo-cólico tanto em animais quanto em humanos (APPLEWHITE; HAWTHORNE; CORNELL, 2001; LAMB; MANTIS, 1998; LEVITT'; BAUER, 1992; MACPHAIL, 2002). Não existe predisposição sexual e racial descritas na literatura quanto à distribuição etária, parece haver um maior acometimento de animais jovens (até um ano de idade), provavelmente relacionado à grande incidência de gastroenterites/enterites infecciosas. Guilford e Strombeck (1996) sugerem que cerca de 80\% dos casos sejam representados por esta faixa etária. A ocorrência em animais idosos geralmente está relacionada a processos neoplásicos, o que também é relatado na literatura médica (BILELLO; PETERSON, 2005; DRASKOVIC et al., 2005; JAREMKO; RAWAT, 2005; KAMO et al., 2005).

Dentre os fatores predisponentes, devem-se ressaltar as alterações de dieta, presença de corpos estranhos, granulomas ou massas gastrointestinais, gastroenterites/enterites infecciosas ou não, parasitismo intestinal e cirurgias prévias, além da forma idiopática, frequente em cães (APPLEWHITE; HAWTHORNE; CORNELL, 2001; GUILFORD; STROMBECK, 1996; MACPHAIL, 2002). Relatos verificam ocorrência de intussuscepções após cirurgias abdominais, como transplantes renais (DU TOIT et al., 1981). A indução de mediadores inflamatórios como prostaglandinas e óxido nítrico é relacionada com quadros de intussuscepções, em razão de sua importância na manutenção da motilidade intestinal (TÜRKYILMAZ et al., 2004).

As prostaglandinas estão relacionadas à contração muscular tanto em intestino delgado quanto grosso. Em geral, prostaglandinas E e F contraem a porção muscular longitudinal, enquanto o subtipo E relaxa e o F contrai a porção circular. Esta substância despertou a atenção de diversos pesquisadores quanto à possibilidade de reverter ou minimizar o íleo paralítico. Entretanto, existe uma variação tecidual da ação das prostaglandinas em musculatura lisa ainda pouco definida (WILSON;

Rev. Acad., Ciênc. Agrár. Ambient., Curitiba, v. 7, n. 3, p. 265-272, jul./set. 2009 
KAYMAKCALAN, 1981). Acredita-se que o subtipo PGE ${ }_{1}$ possa diminuir a motilidade de intestino delgado em cães (SHEHADEH; PRICE; JACOBSON, 1969).

$\mathrm{O}$ óxido nítrico (NO), inicialmente identificado como um fator relaxante derivado do endotélio, tem sido reconhecido como importante neurotransmissor inibitório não adrenérgico, não colinérgico no intestino, interferindo na motilidade e contração muscular (SUNG et al., 2006; WANG et al., 2000). A enzima óxido nítrico sintetase (NOS), responsável pela síntese de NO, apresentam três isoformas: indutível (iNOS), endotelial (eNOS) e neuronal (nNOS). As nNOS, expressas constitutivamente em baixos níveis, são encontradas nos plexos mioentéricos. As indutíveis, usualmente ausentes, podem ser expressas em situações de inflamações e endotoxemia bacteriana (WANG et al., 2000). Acredita-se que o NO participe em diversas enteropatias que cursem com alterações motoras, uma vez que neurônios inibitórios não adrenérgicos não colinérgicos são reguladores da fase de relaxamento do peristaltismo (SUNG et al., 2006; WANG et al., 2000). Na vigência de processos inflamatórios, tem-se a elevação sistêmica de NO e NOS associada ao aumento de demais mediadores inflamatórios, o que pode resultar em alterações de motilidade e aumento da incidência de intussuscepções. A administração de inibidores da via cicloxigenase pode contribuir para a normalização da motilidade intestinal, bem como à utilização de inibidores específicos da enzima óxido nítrico sintetase (SUNG et al., 2006; TÜRKYILMAZ et al., 2004; WANG et al., 2000).

As intoxicações por carbamatos também devem ser consideradas como fatores desencadeantes da intussuscepção. Acredita-se que esta ocorrência esteja relacionada à hipermotilidade gastrointestinal observada em quadros de intoxicações, nos quais ocorre maior estimulação de receptores muscarínicos parassimpáticos (CORFIELD et al., 2008).

\section{SINAIS CLÍNICOS}

Diversos são os sinais clínicos que cursam com esta enfermidade, desde alterações inespecíficas como anorexia, disorexia, depressão, letargia, perda de peso, êmese, diarreia por vezes hemorrágica, sensibilidade e distensão abdominal, estes últimos resultantes da obstrução intestinal, supercrescimento bacteriano, isquemia e infarto da porção acometida, além de peritonite focal ou difusa (ARONSON; BROCKMAN; BROWN, 2000; GUILFORD; STROMBECK, 1996; LAMB; MANTIS, 1998; MACPHAIL, 2002).

A presença de prolapso intestinal atrai a atenção do proprietário e pode facilitar o diagnóstico imediato da afecção. Entretanto, a não diferenciação entre prolapso retal verdadeiro e intussuscepção prolapsada pode comprometer a vida do paciente. Em se tratando de intussuscepção prolapsada, o tratamento correto inclui intervenção cirúrgica para correção intra-abdominal da afecção e não apenas a reintrodução do segmento exteriorizado. Se isto ocorrer, a manutenção do segmento invaginado irá levar ao quadro de isquemia prolongada, necrose, ruptura intestinal, seguido de choque séptico e óbito (CASTRO, 2003; OLIVEIRA; CASTRO; MATERA, 2007).

\section{DIAGNÓSTICO}

Muitos dos quadros de intussuscepção podem ser diagnosticados por meio da palpação abdominal, localizando e delimitando estrutura tubular firme, que deve ser diferenciada de conteúdo fecal ou corpos estranhos (GUILFORD; STROMBECK, 1996). Em estudo realizado com nove gatos e 36 cães, 53\% dos animais apresentaram palpação abdominal compatível com o quadro (LEVITT; BAUER, 1992). Para o diagnóstico definitivo, pode-se valer do uso de técnicas de imagem, das quais o ultrassom apresenta maior acurácia, sensibilidade e especificidade. $\mathrm{O}$ raio-X simples e contrastado, endoscopia e colonoscopia também apresentam utilidade. Dos exames radiográficos contrastados, utiliza-se com maior frequência o enema de bário pela localização preferencial das porções acometidas. O trânsito gastrointestinal pode fornecer o mesmo resultado necessitando de maior tempo de exame. 
$\mathrm{Na}$ avaliação radiográfica, frequentemente encontra-se anormalidade da distribuição de alças no abdômen, dilatação intestinal, presença de variável quantidade de líquido e gás em região cranial a obstrução, até a observação de estrutura de radiopacidade água (intussuscepção propriamente dita). No exame contrastado, podem-se observar dilatações intestinais, além da dificuldade ou impossibilidade da progressão do marcador.

As características ultrassonográficas normalmente ressaltadas são múltiplas linhas paralelas em cortes longitudinais e múltiplos anéis concêntricos hiperecoicos e hipoecoicos ao redor de centro hiperecoico em cortes transversais, comumente correlacionados às imagens de alvos ou olhos de bois (LAMB; MANTIS, 1998; PATSIKAS et al., 2003; PATSIKAS et al., 2004; PATSIKAS et al., 2005). São citadas como vantagens da técnica ultrassonográfica em relação à radiográfica a ausência de exposição à radiação, menor tempo de exame, avaliação de estruturas adjacentes como linfonodos e, de forma mais importante, avaliação da viabilidade intestinal e redutibilidade da intussuscepção com o auxílio do modo Doppler (LAMB; MANTIS, 1998; PATSIKAS et al., 2005). Seu uso tem sido extensamente descrito em humanos, nos quais são utilizadas com muita frequência técnicas de redução pneumática ou hidrostática, sem a necessidade de submeter o paciente à intervenção cirúrgica. Para os animais, esta contribuição mostra-se de grande valia para o planejamento cirúrgico e prognóstico. A ausência de fluxo sanguíneo ao exame está intimamente relacionada com impossibilidade na redução da intussuscepção, presença de aderências, desvitalização de tecido e perfurações. Patsikas et al. (2005) avaliaram, pelo modo Doppler, 15 animais jovens da espécie canina com quadro de intussuscepção. Três animais não apresentavam fluxo sanguíneo nas intussuscepções que, ao procedimento cirúrgico, mostraram-se irredutíveis. Dos animais com fluxo preservado, 75\% tiveram suas lesões reduzidas manualmente e apenas três necessitaram de enterectomias.

\section{TRATAMENTO}

$\mathrm{Na}$ medicina veterinária, o tratamento de escolha ainda é o cirúrgico, no qual podem ser realizadas técnicas de redução manual, ressecção da porção acometida associada à enteroanastomose e uso de enteroplicações (BROWN, 2003; HEDLUNG, 2002; MACPHAIL, 2002). A escolha da técnica depende da viabilidade e do grau de lesão intestinal (MACPHAIL, 2002).

Os quadros recentes podem ser reduzidos mecanicamente, por meio de leve tração das porções adjacentes às acometidas (ARONSON; BROCKMAN; BROWN, 2000). Em contrapartida, intussuscepções crônicas frequentemente necessitam de enterectomias por causa das lesões irreversíveis do tecido ou irredutibilidade da intussuscepção. Applewhite, Hawthorne e Cornell (2001) relataram necessidade de ressecção e enteroanastomose em 27 dos 35 casos avaliados (77\%). O emprego de técnicas fechadas de redução, como a pneumática ou a hidrostática, fica limitado na veterinária pela maior incidência de casos crônicos. Abasiyanik et al. (1997) realizaram a técnica de redução pneumática com auxílio de laparoscopia em 27 cães, obtendo sucesso em $96 \%$ e taxa de 4\% de recorrência e perfuração intestinal. O intervalo de tempo entre o desenvolvimento da intussuscepção até a intervenção variou apenas de três a cinco dias. A redução espontânea, principalmente após o uso de medicação préanestésica é pouco frequente, porém possível de ocorrer. Recomenda-se a palpação abdominal e localização da intussuscepção imediatamente antes do início da cirurgia, a fim de se evitar procedimentos desnecessários (PATSIKAS; PAPAZOGLOU; ADAMAMA-MORAITOU, 2008).

\section{COMPLICAÇÕES}

As complicações mais observadas no pós-operatório são recorrência do quadro, íleo paralítico, deiscência da anastomose, obstrução intestinal, peritonite e síndrome do intestino curto, sendo as duas primeiras as mais frequentes (APPLEWHITE; HAWTHORNE; CORNELL, 2001; MACPHAIL, 2002). A literatura cita cerca de 11 a 20\% de recorrência, sendo maior a recidiva em 
casos tratados com a redução manual (25\%) quando comparado com animais que sofreram enterectomia da porção acometida (19\%).

Estudos relacionam diminuição de reincidências em animais tratados com derivados de opioides, durante o trans ou pós-operatório. O mecanismo não está totalmente esclarecido, porém discute-se um possível aumento do tônus muscular intestinal (APPLEWHITE; HAWTHORNE; CORNELL, 2001). Mcnaulty, Southard e Belzer (1989) descrevem redução de 17\% para 3\% de intussuscepções em animais previamente submetidos a transplante renal experimental com o uso de morfina, o que está de acordo com o resultado encontrado por Klinger, Cooper e McCabe (1990) com o uso de butorfanol. Por outro lado, o largo emprego de opioides pode contribuir para o retardo do trânsito gastrointestinal e para o desenvolvimento ou agravamento do íleo paralítico.

Procedimentos cirúrgicos recomendados para a prevenção de recidivas baseiam-se na criação de aderências permanentes entre as curvas intestinais, chamadas de enteroplicações (ARONSON; BROCKMAN; BROWN, 2000; NASH; BELLENGER, 1998). A literatura é controversa com relação à eficácia dessas técnicas. Alguns autores citam benefícios enquanto outros mostram não haver diferença estatisticamente significante entre os grupos testados (APPLEWHITE; HAWTHORNE; CORNELL, 2001; ARONSON; BROCKMAN; BROWN, 2000; KYLES; SCHNEIDER; CLARE, 1998; LEVITT; BAUER, 1992; LEWIS; ELLISON, 1987; MACPHAIL, 2002). Além disso, complicações decorrentes desta técnica, como estrangulamento e obstrução intestinal, tornam necessária nova intervenção cirúrgica para correção da afecção desenvolvida (APPLEWHITE; HAWTHORNE; CORNELL, 2001; KYLES; SCHNEIDER; CLARE, 1998; MACPHAIL, 2002).

Diversos estudos apresentam o íleo paralítico pós-operatório como condição relativamente comum em cirurgias abdominais, principalmente nas intestinais. Trata-se de uma alteração transitória e reversível, na qual ocorre disfunção ou inibição da motilidade intestinal. Esta anormalidade da motilidade pode predispor ao supercrescimento bacteriano, que exacerba os sinais clínicos já observados (WASHABAU, 2003). Pode acometer tanto estômago, intestino delgado quanto grosso (WASHABAU, 2003). Clinicamente, observa-se anorexia, êmese, distensão abdominal e sensibilidade dolorosa difusa relacionada ao acúmulo de gases pela hipomotilidade intestinal, além de alterações do trânsito intestinal como aquesia ou episódios diarreicos. Quando afetados de forma crônica, os animais podem apresentar mudanças da condição corpórea, como perda de peso. Sua patogenia ainda é discutida, porém, estudos revelam possível dissociação eletromecânica influenciada por fatores humorais, neurais e metabólicos ainda não claramente definidos. Isto parece ser resultante da ativação de um reflexo neural inibitório por inervação simpática e ativação de processos inflamatórios, nos quais o óxido nítrico está fortemente envolvido, porém sem via de ação totalmente esclarecida (TÜRKYILMAZ et al., 2004). A severidade da injúria é diretamente proporcional à magnitude da resposta inflamatória e ao grau de acometimento clínico (KALFF et al., 1998). O grau de lesão tecidual e a manipulação cirúrgica podem ser relacionados à intensidade da estimulação simpática e, consequentemente, à estase intestinal pós-operatória (BROWN, 2003).

A resolução do quadro de íleo adinâmico ocorre com a restauração da função intestinal, o que pode ser alcançado apenas com a eliminação da injúria intestinal. Em alguns casos, faz-se necessário o uso de agentes procinéticos e reguladores intestinais sintéticos ou naturais.

Semelhante às apresentações clínicas do íleo paralítico, as disfunções motoras decorrentes de lesões nervosas são relatadas, porém de maneira permanente. O funcionamento errôneo do aparelho gastrointestinal acaba por prejudicar processos de digestão, absorção e eliminação. É relatada a ocorrência de acúmulo de conteúdo fecal resultando em processos obstrutivos e alterações na absorção de nutrientes, influenciando na condição corporal e qualidade de vida do paciente.

Estudos recentes buscam utilizar inibidores de NO como bloqueadores desta cascata inflamatória e evitando o desenvolvimento do íleo adinâmico. Uenoyama et al. (2004) utilizaram sulfato de S-methylisothiourea (SMT), inibidor da óxido nítrico sintetase, em animais com injúrias intestinais iatrogênicas e encontraram alterações compatíveis com a literatura no aumento do tempo de trânsito intestinal, quantidade de nitrito e nitrato produzidos e expressão de RNAs de NO quando comparado com grupo controle. 
Outra possível complicação é a síndrome do intestino curto, distúrbio relacionado às extensas ressecções intestinais, principalmente na região referente ao cólon. Sabe-se que o cão é capaz de manter funções digestivas normais com cerca de 30 a 40 centímetros de intestino delgado (BARKER; VAN DREUMAL; PALMER, 1993). Entretanto, nem sempre essas adaptações são suficientes para a homeostase intestinal, o que resulta em má digestão, má absorção, perda de peso e diarreias intermitentes ou contínuas. Nestes casos, os animais necessitam de suporte nutricional mais intenso sob a forma parenteral durante a fase inicial. O uso de fibras e medicamentos que retardam o esvaziamento gastrointestinal pode ser associado a alimentos de alta digestibilidade. Opções cirúrgicas têm sido utilizadas na medicina, como a criação de válvulas intestinais e esfíncteres artificiais, alongamento e afilamento intestinal ou o transplante de porções intestinais (GUILFORD; STROMBECK, 1996; RASSLAN, 2007a, b, c).

Rasslan (2007b) descreve a ocorrência de inúmeras alterações gastrointestinais após ressecções intestinais extensas, com o intuito de adaptar o organismo ao novo comprimento do órgão. Sabe-se que o encurtamento do intestino acaba por gerar menor tempo de trânsito alimentar, principalmente se há o envolvimento da válvula íleo-ceco-colica. Além de alterações microscópicas, como a hiperplasia e hipertrofia de segmentos intestinais remanescentes e "delgadização" do intestino grosso, ocorrem alterações do comportamento do trânsito alimentar com estase gástrica e retardo na eliminação de ingestas/bolo fecal através de alterações de motilidade (RASSLAN, 2007a, b). Esta alteração de motilidade tem por finalidade propiciar maior tempo de contato entre intestino e conteúdo luminal, a fim de permitir absorção adequada de nutrientes e evitar o desenvolvimento de síndromes de má absorção, espoliação metabólica, desnutrição, anemia e perda de massa muscular e gordurosa. Presume-se que o retardo no esvaziamento gástrico ocorra por ação dos freios intestinais, acionados mais precoce e intensamente em decorrência do intestino reduzido e da concentração aumentada de nutrientes no íleo. Quanto ao trânsito intestinal, o tempo é mais curto porque o segmento remanescente é menor, mas os movimentos peristálticos são mais lentos, estabelecendo um mecanismo compensatório (RASSLAN, 2007a, b).

Esta revisão tem como objetivo contribuir para o conhecimento de uma afecção frequente e importante na clínica de pequenos animais, nem sempre diagnosticada prontamente. $O$ diagnóstico precoce, associado à correção cirúrgica, é fundamental para o sucesso do tratamento. Quando corretamente identificada e tratada, a intussuscepção apresenta bom prognóstico e baixo índice de recidiva.

\section{REFERÊNCIAS}

ABASIYANIK, A. et al. Laparoscopic-assisted pneumatic reduction of intussusception. Journal of Pediatric Surgery, London, v. 32, n. 8, p. 1147-1148, 1997.

APPLEWHITE, A. A.; HAWTHORNE, J. C.; CORNELL, K. K. Complications of enteroplication for the prevention of intussusception recurrence in dogs: 35 cases (1989-1999). Journal of the American Veterinary Medical Association, Schaumburg, v. 219, n. 10, p. 1415-1418, 2001.

ARONSON, L. R.; BROCKMAN, D. J.; BROWN, D. C. Gastrointestinal emergencies. Veterinary Clinics of North America: Small Animal Practice, Amsterdam, v. 30, n. 3, p. 555-579, 2000.

BARKER, I. K.; VAN DREUMAL, A. A.; PALMER, N. The alimentary system. In: JUBB, V. F.; KENNEDY, P. C.; PALMER, N. Pathology of domestic animal. London: Academic Press, 1993. p. 101.

BILELLO, J. F.; PETERSON, W. M. Retrograde jejunojejunal intussusception secondary to metastatic melanoma. Mayo Clinic Proceedings, Rochester, v. 80, n. 8, p. 1098, 2005.

BROWN, D. C. Small intestines. In: SLATTER, D. Textbook of small animal surgery. Philadelphia: Saunders, 2003. p. 644-664. 
CASTRO, P. F. Prolapso ou intussuscepção de reto: reduzir ou amputar, eis a questão! Brazilian Journal of Veterinary Research and Animal Science, São Paulo, v. 40, p. 137-138, 2003.

CORFIELD, G. S. et al. Intussusception following methiocarb toxicity in three dogs. Journal of Veterinary Emergency and Critical Care, San Antonio, v. 18, n. 1, p. 68-74, 2008.

DRASKOVIC, M. et al. Jejuno-jejunal intussusception in adults secondary to submucosal leiomyoma. Medicinski Pregled, Belgrado, v. 58, n. 7-8, p. 405-409, 2005.

DU TOIT, D. et al. Canine intestinal intussusception following renal and pancreatic transplantation. Veterinary Records, London, v. 108, n. 2, p. 34-35, 1981.

GUILFORD, W. G.; STROMBECK, D. R. Intestinal obstruction, pseudo-obstruction and foreign bodies. In: GUILFORD, W. G. et al. (Ed.). Strombeck's small animal gastroenterology. 3rd ed. Philadelphia: Saunders, 1996. p. 487-502.

HEDLUNG, C. S. Cirurgia do intestino delgado. In: FOSSUM, T. W. (Ed.). Cirurgia de pequenos animais. São Paulo: Roca, 2002. p. 322-349.

JAREMKO, J. L.; RAWAT, B. Colo-colonic intussusception caused by a solitary Peutz-Jeghers polyp. The British Journal of Radiology, London, v. 78, n. 935, p. 1047-1049, 2005.

KALFF, J. C. et al. Surgical manipulation of the gut elicits an intestinal muscularis inflammatory response resulting in post-surgical íleus. Annals of Surgery, Alphen aan den Rijn, v. 228, n. 5, p. 652-663, 1998.

KAMO, R. et al. A case of ileoileal intussusception caused by metastatic pedunculated tumor of cutaneous angiosarcoma. The Journal of Dermatology, Tokyo, v. 32, n. 8, p. 638-640, 2005.

KLINGER, M.; COOPER, J.; MCCABE, R. The use of butorphanol tartrate for the prevention of canine intussusception following renal transplantation. Journal of Investigative Surgery, London, v. 3, n. 3, p. 229-233, 1990.

KYLES, E. A.; SCHNEIDER, T. A.; CLARE, A. Foreign body intestinal perforation and intraabdominal abscess formation as a complication of enteroplication in a dog. The Veterinary Record, London, v. 143, n. 4, p. 143-144, 1998.

LAMB, C. R.; MANTIS, P. Ultrasonographic features of intestinal intussusception in 10 dogs. Journal of Small Animal Practice, Gloucester, v. 39, n. 9, p. 437-441, 1998.

LEVITT, L.; BAUER, M. S. Intussusception in dogs and cats: a review of thirty-six cases. The Canadian Veterinary Journal, Gloucester, v. 33, n. 10, p. 660-664, 1992.

LEWIS, D. D.; ELLISON, G. W. Intussusception in dogs and cats. Compendium on Continuing Education for the Practicing Veterinarian, Schaumburg, v. 9, n. 5, p. 523-534, 1987.

MACNAULTY, J. F.; SOUTHARD, J. H.; BELZER, F. O. Prevention of postoperative intestinal intussusception by prophylactic morphine administration in dogs used organ transplantation research. Surgery, Philadelphia, v. 105, n. 4, p. 494-495, 1989.

MACPHAIL, C. Gastrointestinal obstruction. Clinical Techniques in Small Animal Practice, New York, v. 17, n. 4, p. 178-183, 2002.

NASH, J. M.; BELLENGER, C. R. Enteroplications in cats, using suture of N-butyl cyanoacrylate adhesive. Research in Veterinary Science, Edinburgh, v. 65, n. 3, p. 253-258, 1998.

OLIVEIRA, L. M.; CASTRO, P. F.; MATERA, J. M. Estudo comparativo de intussuscepções prolapsadas em cães durante o período de 2000 a 2005. Pesquisa Veterinária Brasileira, Rio de Janeiro, v. 27, p. 60-61, 2007. Suplemento. 
PATSIKAS, M. N. et al. Ultrasonographic signs of intestinal intussusception associated with acute enteritis or gastroenteritis in 19 young dogs. Journal of the American Animal Hospital Association, Lakewood, v. 39, n. 1, p. 57-66, 2003.

. Normal and abnormal ultrasonographic findings that mimic small intestinal intussusception in the dog. Journal of the American Animal Hospital Association, Lakewood, v. 40, n. 2, p. 147-151, 2004.

Doppler untrasonography in prediction of the reducibility of intussuscepted bowel in 15 young dogs. Veterinary Radiology \& Ultrasound, Raleigh, v. 46, n. 4, p. 313-316, 2005.

PATSIKAS, M. N.; PAPAZOGLOU, L. G.; ADAMAMA-MORAITOU, K. Spontaneous reduction of intestinal intussusception in five young dogs. Journal of the American Animal Hospital Association, Lakewood, v. 44, n. 1, p. 41-47, 2008.

RASSLAN, S. Efeitos secundários das ressecções intestinais extensas. In: Ressecções intestinais extensas e síndrome do intestino curto. São Paulo: Atheneu, 2007a. p. 19-34.

Motilidade intestinal após enterectomias extensas. In: Ressecções intestinais extensas e síndrome do intestino curto. São Paulo: Atheneu, 2007b. p. 35-38.

Tratamento cirúrgico complementar. In: Ressecções intestinais extensas e síndrome do intestino curto. São Paulo: Atheneu, 2007c. p. 51-58.

SHEHADEH, Z.; PRICE, W. E.; JACOBSON, E. D. Effects of vasoactive agents on intestinal blood flow and motility in the dog. The American Journal of Physiology, Stanford, v. 216, n. 2, p. 386-392, 1969.

SUNG, T. S. et al. Alteration of nitrergic neuromuscular transmission as a result of acute experimental colitis in rat. Journal of Veterinary Science, Seoul, v. 7, n. 2, p. 143-150, 2006.

TÜRKYILMAZ, Z. et al. Role of nitric oxide and cyclooxygenase pathway in lipopolysaccharideinduced intussusception. Pediatric Surgery International, Berlin, v. 20, n. 8, p. 598-601, 2004.

UENOYAMA, S. et al. Improvement of intestinal motility using S-methylisothiourea in postoperative ileus. American Journal of Surgery, Belle Mead, v. 187, n. 1, p. 93-97, 2004.

WANG, Z. Q. et al. Involvement of endogenous nitric oxide and c-kit-expressing cells in chronic intestinal pseudo-obstruction. Journal of Pediatric Surgery, London, v. 35, n. 4, p. 539-544, 2000.

WASHABAU, R. J. Gastrointestinal motility disorders and gastrointestinal prokinetic therapy. The Veterinary Clinics of North America: Small Animal Practice, Amsterdam, v. 33, n. 5, p. $1007-$ 1028, 2003.

WILSON, D. E.; KAYMAKCALAN, H. Prostaglandins: gastrointestinal effects and peptic ulcer disease. The Medical Clinics of North America, Philadelphia, v. 65, n. 4, p. 773-787, 1981.

Recebido: 05/11/2008

Received: 11/05/2008

Aprovado: 20/01/2009

Approved: 01/20/2009 